12 King's Bench Walk

Follow Daniel on Twitter: @DanielSokol9 Cite this as: BMJ 2021;375:n2670 http://dx.doi.org/10.1136/bmj.n2670 Published: 02 November 2021

\title{
Covid-19 vaccination should be mandatory for healthcare workers
}

\section{Daniel Sokol medical ethicist and barrister?}

The Royal College of Physicians and the Royal Pharmaceutical Society have spoken out against mandatory covid vaccinations for healthcare staff. The Royal College of Nursing has "significant concerns” about it. On 14 October 2021, the Nuffield Council on Bioethics called for more evidence of the need for mandatory vaccination before resorting to coercion. ${ }^{1}$ In contrast, countries such as France, Greece, Italy, and Hungary have already made covid vaccination mandatory for healthcare workers and last week the UK's secretary of state for health and social care, Sajid Javid, said that he was "leaning towards" compulsory vaccination. So who is right?

As this pandemic has shown, the problem with waiting for more information is that delaying action costs lives. The UK is now an outlier in Europe with a disproportionately high number of new cases per day. France's seven day average of new cases on 27 October 2021 was 5,459, with 30 deaths a day. Italy's was 3,783, with 38 deaths a day. The UK's figure was 43 916, with 144 deaths a day. ${ }^{2}$ The latest $R$ value for England is now 1.1 to 1.3 , which means that on average every 10 people infected will infect 11 to 13 others. ${ }^{3}$

According to Javid, there are around 100000 unvaccinated NHS staff. These staff are far more likely to transmit the virus than their vaccinated counterparts. Researchers at the University of Melbourne have estimated that there is a 20-fold reduction in risk of contracting covid when in contact with a vaccinated person as compared to an unvaccinated person. ${ }^{4}$ Few patients would want to be treated by a doctor or nurse who may be in contact with dozens of sick patients every day, but remains unvaccinated against covid.

It is trite that healthcare workers who come in contact with patients have an ethical obligation to be vaccinated. The first rule of the General Medical Council's Duties of a Doctor is "Make the care of your patient your first concern." The GMC also instructs doctors to be "immunised against common serious communicable diseases (unless otherwise contraindicated)." In short, an unvaccinated healthcare worker could be disciplined or even lose their job. Making vaccination compulsory is hardly more coercive. It would add a legal obligation to the existing ethical one.

Judging from the experience with care home workers, for whom vaccination will become compulsory from 11 November 2021, implementing this policy should result in a boost in uptake. ${ }^{5}$ There have been some reports of unvaccinated care workers quitting their jobs over compulsory covid vaccines, but the full impact of the new law on staff levels is not yet known. ${ }^{6}$
The potential impact on the workforce is clearly a source of concern to NHS trust leaders. A survey of 172 such leaders, conducted by NHS Providers from August to October 2021, showed that 58\% supported mandatory covid vaccinations for staff, but that over $90 \%$ were concerned about the potential for staffing gaps in the NHS and social care arising from this requirement.

A look at what has happened in France is informative. The new law on mandatory vaccination for healthcare workers led to a massive boost in vaccination rates, from $60 \%$ in July (when the new requirement was announced) to over $99 \%$ in October. The French health minister, Oliver Véran, stated on 13 October 2021 that no more than $0.1 \%$ of healthcare staff who refused to be vaccinated have left their jobs. ${ }^{7}$

Is mandatory vaccination an infringement on the individual freedom of healthcare workers?

Absolutely, but this relatively minor infringement is justified for the sake of protecting the health of others. Might compulsion lead to some healthcare workers leaving the profession, increasing the pressure on existing staff? Possibly, but infecting others or becoming ill oneself also adds to the burden and disruption.

Remember also that, even without compulsion, these healthcare workers could be subject to disciplinary action or termination of employment, effectively removing them from the medical workforce.

Some colleagues have called for a detailed evaluation of why some healthcare staff refuse the vaccine and to explore gentler ways to persuade. This sounds reasonable, but the problem is that conducting this research takes time, probably months. Further, there is no guarantee that the results will yield much of practical value. They may demonstrate a wide range of reasons for refusing the vaccine and ambivalence about remaining in the profession if encouraged or compelled to have the vaccine. Such equivocal results may provide little of substance for decision-makers. It is easy to forget that there is a real human cost to waiting for results, measured in avoidable illness and deaths. Right now, there are likely to be unvaccinated doctors, nurses, and other clinicians who, in breach of medical ethics, are seeing patients and putting them at needlessly high risk of contracting a serious disease.

There comes a time when a decision has to be made, even with imperfect or incomplete information. That time is now. Like care home workers and their counterparts in many European countries, in my opinion, healthcare staff in the UK, unless exempt, should be subject to mandatory covid vaccination to protect the lives and health of those they treat. 


\section{OPINION}

Provenance and peer review: not commissioned, not peer reviewed

1 Mandatory vaccinations for health and social care workers https://www.nuffield-

bioethics.org/news/mandatory-vaccinations-for-health-and-social-care-workers-nuffield-council-

on-bioethics-urges-government-to-gather-more-evidence-and-explore-other-options-more-

thoroughly-before-introducing-coercive-measures

2 Covid-19 Data Repository by the Center for Systems Science and Engineering at Johns Hopkins University https://www.arcgis.com/apps/dashboards/bda7594740fd40299423467b48e9ecf6

3 The R value and growth rate https://www.gov.uk/guidance/the-r-value-and-growth-rate

4 Your unvaccinated friend is roughly 20 times more likely to give you COVID https://theconversation.com/your-unvaccinated-friend-is-roughly-20-times-more-likely-to-give-you-covid-170448

5 Sajid Javid leaning towards mandatory vaccination, The Guardian, https://www.theguardian.com/world/2021/oct/25/sajid-javid-leaning-towards-mandatory-covid-jab-nhs-staff

6 Unvaccinated care workers in Portsmouth quit https:/www.hampshirelive.news/news/hampshirenews/unvaccinated-care-workers-portsmouth-quit-5930240

7 Coronavirus: Seuls 0,6\% des personnels soignants ne sont pas vaccinés, affirme Olivier Véran https://www.20minutes.fr/sante/3147215-20211013-coronavirus-seuls-06-personnels-soignantsvaccines-affirme-olivier-veran 